\title{
Automatic Classification of Stellar Spectra used Neural Network
}

\author{
Liangping Tu, Fuchao Wu \\ Institute of Automation, \\ Chinese Academy of Sciences \\ Zhongguancun East Road 95, Beijing 100190, China \\ lptu@nlpr.ia.ac.cn, fcwu@nlpr.ia.ac.cn
}

\author{
Ali Luo, Jiannan Zhang \\ National Astronomical Observatories, \\ Chinese Academy of Sciences \\ Datun Road 20, Beijing 100012, China \\ lal@lamost.org,jnzhang@lamost.org
}

\begin{abstract}
Large field spectra survey such as LAMOST will collect enormous amounts of spectra data, and these mission will require reliable and automated methods for classifying the large number of spectra data. In this paper, we present an automatic classification system of stellar spectra used neural network, it can achieve the so called MK spectral classification scheme, which is a two parameter classification, spectral type and luminosity class. The system is composed of 2 functional units, namely stellar spectra preprocessing and the classification of spectral type and luminosity class. Experiments show that the observation stellar spectra can be classed accurately in the resolution of LAMOST spectra.
\end{abstract}

\section{Introduction}

The Large Sky Area Multi-Object Fiber Spectroscopic Telescope (LAMOST) project is one of the National Key Scientific Projects undertaken by the Chinese Academy of Science. After the completion of LAMOST project at the middle of 2008, the scheduled Galaxy survey will collect almost $10^{7}$ spectra of stars. Such voluminous spectra data urgently demand to explore automatic recognition methods, which is important for the astronomy research. The recognition and classification of spectra are very difficult problems. The spectral types and luminosity classes represent different properties of star. Spectral type is a token of the effective temperature, while the luminosity indicates the unit intensity of a star. This two dimensional classification provides fundamental physics measurement of stars. But for LAMOST and other similar spectra survey projects, low-resolution stellar spectra are difficult to determine the luminosity class and spectral sub-type. Many works have been tried on the spectral type recognition, which is comparative easy, while a few work on luminosity type recognition. We have used a stratified approach [5] which combined the non-parameter estimation algorithm and PLS regression to determine spectral types (main-type and sub-type) and luminosity classes of stars. At present, the ANN (artificial neural network)have been used in analyzing astronomical spectra, for example, Bailer-Jones [1] have used ANN to determine the stellar physical parameters for synthetic spectra, and Meifang Zhao [6] have used Radial Basis Function network to identify QSOs and Galaxies, etc.

Since the spectral type and luminosity class of a star have relation with its different properties, we used two BP network which have different configuration to determine the spectral type and luminosity class respectively. In this work, we propose a system for stellar spectra classification. As shown in figure 1, the system consists of two units : 1)Stellar spectra preprocess, include continuum spectrum extraction and spectral normalizing. 2)Determine the spectra type and luminosity class, by feeding input the continuum signal and normalized spectrum signal respectively into Net1 and Net2 which have been trained and output the result. In the following, a description for each unit is given and experiments are reported.

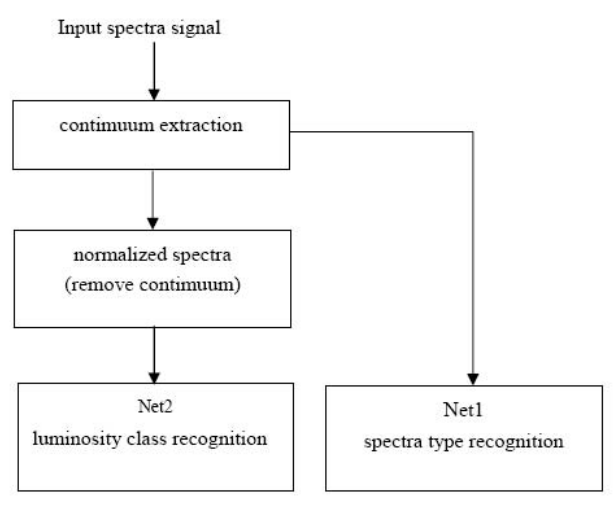

Figure 1. System block diagram 


\section{Stellar spectra preprocess}

In general, stellar spectra are classified into seven main-type: O, B, A, F, G, K, and M. The seven main-type sequence represents the temperature variation: from $\mathrm{O}$ to $\mathrm{M}$ type, the temperature decreases from $50000 \mathrm{~K}$ to $3000 \mathrm{~K}$. As shown in figure 2, the seven main-type stars are different in the shape of spectra, especially the shape of the continuum component. Each main-type is classified into ten subtypes, marked with ten numbers of $0 \sim 9$. The stellar subtype also represents the temperature decreasing from subclass 0 to subclass 9 . But the difference of successive subtype star is very small, that even for astronomers some neighbor subtype spectra are hard to be distinguished. Figure 3 illustrates different subclasses spectra of A type star. Luminosity is another property of star. Usually, the luminosity of a star belongs to one of five classes, marked by I, II, III, IV and V respectively. Figure 4 illustrates the five luminosity classes of spectra of $\mathrm{F}$ type stars.

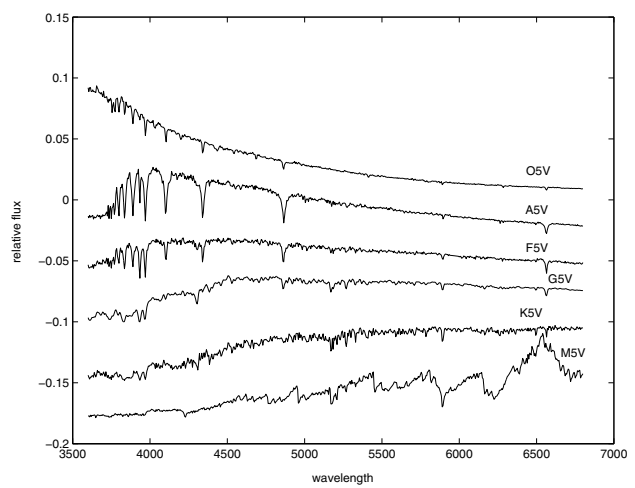

Figure 2. Spectra of $O \sim M$ type star

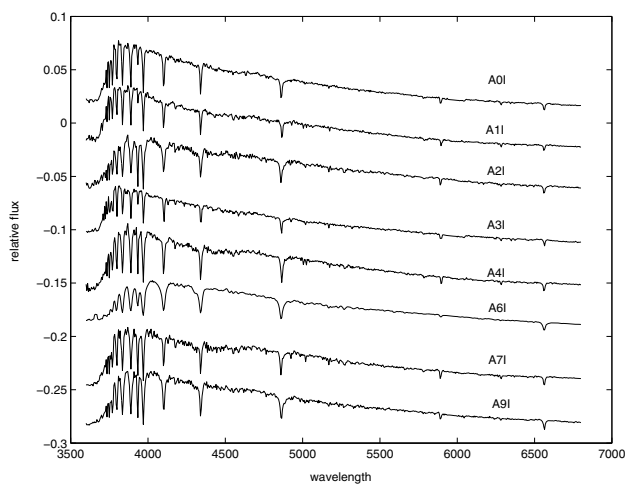

Figure 3. Spectra of A type star with subtype of $0 \sim 9$

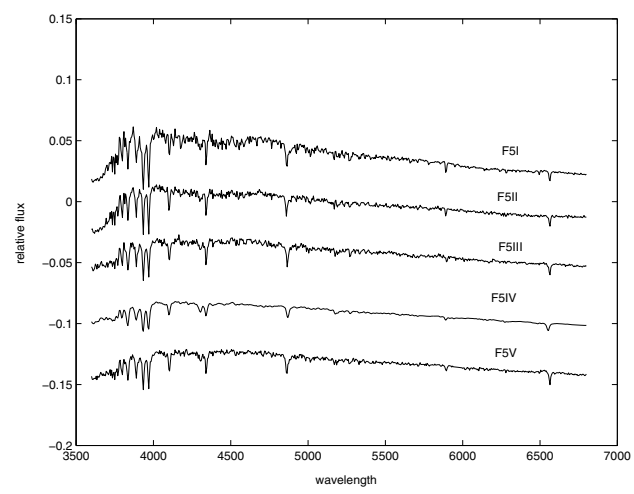

Figure 4. Spectra of F5 type star with luminosity of I to $V$

Because the spectral lines shape partially represents the luminosity and the continuum spectrum represents the spectral type, we used the continuum spectrum and normalized spectrum to determine spectral type and luminosity class respectively. The procedure of continuum normalization has two steps: 1)Using wavelet method to extract the high frequency components of spectra, which are mainly the spectral lines and noise, then reconstructing the low frequency component, which is mainly the continuum component. 2)Fitting the reconstructed component by polynomial to get the continuum, and then removing the continuum from original spectrum to get the normalized spectra. Figure 5 illustrates an A0V spectra, its continuum spectrum and normalized spectrum.
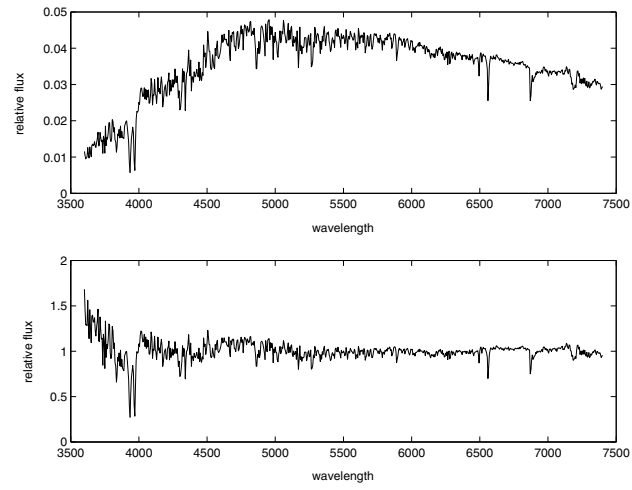

Figure 5. The original spectra and the continuum normalized spectra 


\section{Network system description}

\subsection{The neural network model}

The neural network is an algorithm which performs a non-linear parameterized mapping between an input vector, $\mathrm{X}$, and an output vector, $\mathrm{y}$. After compared RBF network and BP network, we chose the BP network with one hidden layer in this work. The output from the hidden layer is the vector $\mathrm{P}$, the elements of which are given by

$$
\begin{gathered}
p_{j}=\operatorname{radbas}\left(\sum_{i} w_{i, j} x_{i}\right) \\
\operatorname{radbas}(n)=\exp \left(-n^{2}\right)
\end{gathered}
$$

These values are then passed through output layer, the output from the network, $y$, is then the weighted sum of these values,

$$
y_{k}=\sum_{j} w_{j, k} p_{j}
$$

the radbas function provides the non-linear capability of the network, and the weights $w_{j, k}$ are its free parameters.

\subsection{Stellar Spectral Type Representation and Classification}

The stellar spectral main-type and subtype are the measurements of temperature, which is the most important factor of the spectral shape. Firstly we label the main-type and subtype with numbers: $O$ is labeled with $1, \mathrm{~B}$ labeled with 2, A labeled with 3, and so on, $M$ labeled with 7; while the ten subtypes are labeled with its marker multiplied by 0.1 , namely the subtype 1 is labeled with 0.1 , subtype 2 is labeled with 0.2. As the result, A0 type star is labeled with the number of 3.0, and F5 type star is labeled with the number of 4.5. In this way, there are 70 numbers in all to labeling the classes of star theoretically. In fact, some types of star are not exist, such as $\mathrm{O} 0 \sim \mathrm{O} 3$.

In this work, we applied the BP network which have the structure 481-i-1 to determine the main-type and subtype. The number of nodes in hidden layer, namely $i$, has been decided by comparing the test error from $i=5$ to $i=50$, and $i=12$ with minimal test error, as is shown in figure 6 . And the input vector is the continuum spectrum, the wavelength covers $3600 \sim 6000 \AA$ with resolution $5 \AA$.

\subsection{Luminosity class representation and classification}

Luminosity of star affects the shape of spectral lines, from class-I to class- $\mathrm{V}$, spectral lines become weaker and

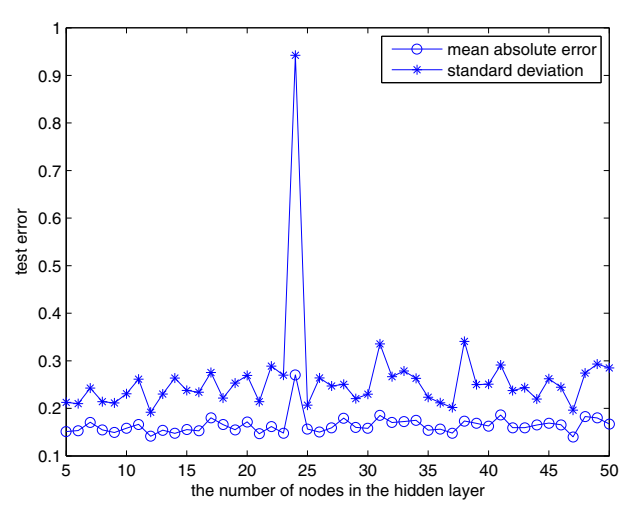

Figure 6. the error of spectral type between original value and test value with different numbers of nodes in the hidden layer

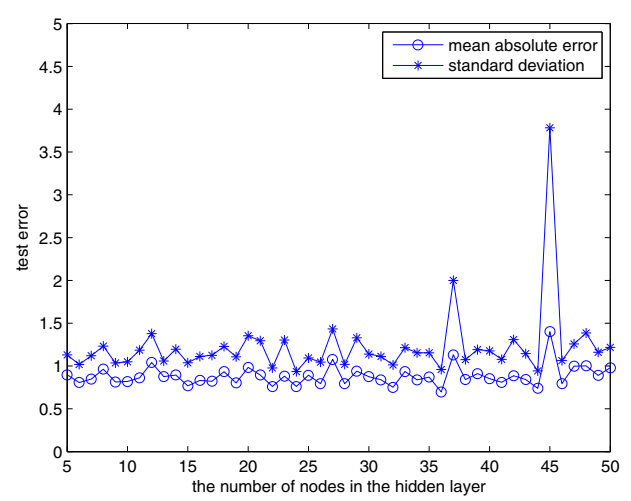

Figure 7. the error of luminosity class between original value and test value with different numbers of nodes in the hidden layer

narrower. Because the shape of spectral lines is also affected by temperature, so the shape of spectral lines is not the measurement only for luminosity. We applied the BP network which have the structure $481-j-1$ to determine the luminosity class of stars, $j=24$ which has been decided by comparing the test error from $j=5$ to $j=50$, as is shown in figure 7. The input vectors are normalized spectra which have been got from 'spectra preprocess'.

\section{Experiment}

In this work, we have carried on experiments with real spectra data from three stellar spectra libraries: 71 spectra from Silva library [4], 129 spectra from Pickles library [3] and 159 spectra from Jacoby library [2]. There are totally 
359 spectra with types ranging from $\mathrm{O} \sim \mathrm{M}$ and luminosity

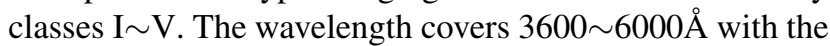
resolution which approximates the resolution of LAMOST. We chose 181 samples for training and 178 samples for testing stochastically. We got the standard deviation of main-type and subtype is $\sigma=0.2259$, and the standard deviation of luminosity class is $\sigma=1.1678$; for the other type stars except for $\mathrm{M}$ type stars, the standard deviation of main-type and subtype is $\sigma=0.1997$, and the standard deviation of luminosity is $\sigma=0.9346$. For the same samples, the result was excelled Zhang's result[5] obviously, in that paper, the standard deviation of spectral type is $\sigma=0.2905$, and the standard deviation of luminosity is $\sigma=1.0453$.

The statistical histogram of residual of main-type and subtype classification and residual of luminosity classification are shown respectively in Figure 8 and Figure 9. And Figure 10 illustrates the result of comparison between the original value and the estimated value of spectra type using our method.

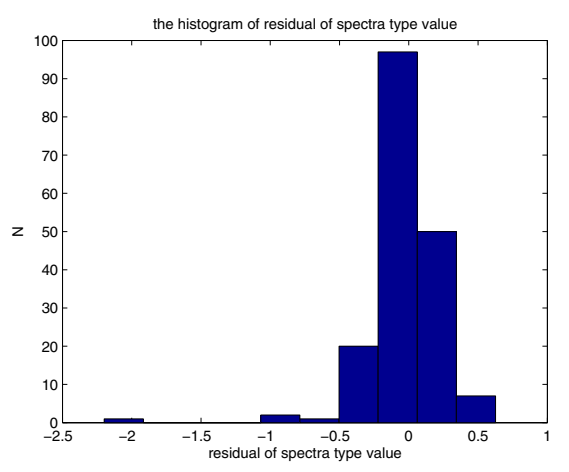

Figure 8. The statistical histogram of residual of spectral type

\section{Conclusion and remarks}

An automatic stellar spectra classification system is proposed in this work. The system realizes two goals: the first is main-type and subtype classification, the second is luminosity classification. Firstly, We preprocessed the primary spectra and got the continuum and normalized spectrum (removed continuum). And then, we took them as input of two BP networks with different structure to determine the spectra type and luminosity class respectively. Experiments showed that our method can obtain a satisfactory result for the low resolution spectra.

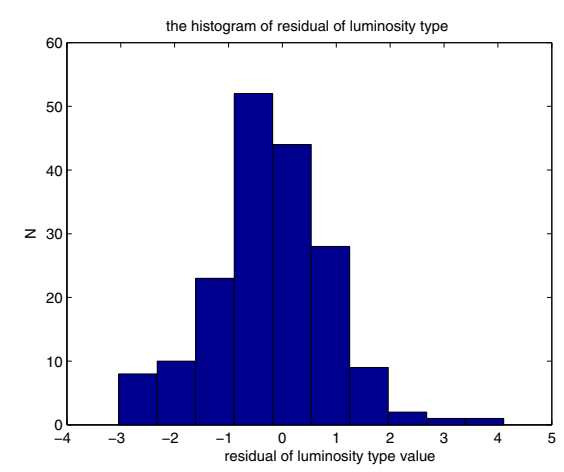

\section{Figure 9. The statistical histogram of residual of luminosity class}

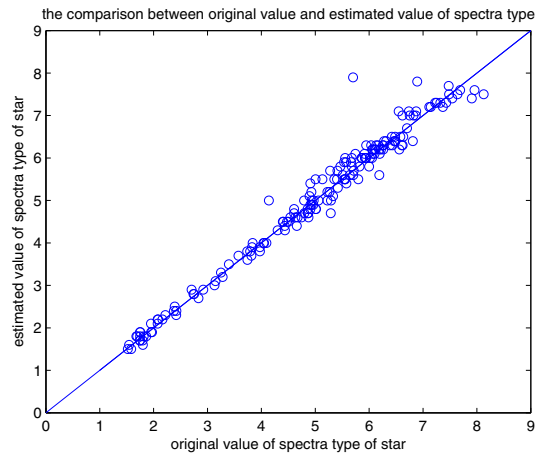

\section{Figure 10. The comparison of original value( $x$ axis) and estimated value(y axis) of the spec- tra type of stars}

The methods in this paper have not been applied in large stellar data set, we believe when we classify large amount of stellar spectrum in the following work, the method should be improved, and we will get more useful statistic result.

Feature extraction has not been used, we think measurement of spectral lines before classify will get more precise result. the spectral lines information such as the width and depth will be introduced into the luminosity classification unit in our next step.

\section{Acknowledgements}

This work was supported by the National Natural Science Foundation of China under grant No.60773040. 


\section{References}

[1] C. Bailer-Jones. Stellar parameters from very low resolution spectra and medium band filters. ASTRONOMY AND ASTROPHYSICS, 357:197-205, 2000.

[2] G. H. Jacoby, D. A. Hunter, and C. A. Christian. A library of stellar spectra. Astrophysical Journal Supplement Series, 56:257-281, 1984.

[3] A. J. Pickles. A stellar spectral flux library: 1150-25000å. Publications of the Astronomical Society of the Pacific, 110:863-878, 1998.

[4] D. R. Silva and M. E. Cornell. A new library of stellar optical spectra. Astrophysical Journal Supplement Series, 81:865$881,1992$.

[5] J. N. zhang, A. L. Luo, and L. P. Tu. A stratified approach for automatic stellar spectra classification. International Congress on Image and Signal Processing, China, 2008.

[6] M. F. zhao, A. L. Luo, F. C. Wu, and Z. Y. Hu. Automated recognition of quasars based on adaptive radial basis function neural networks. Spectroscopy and Spectral Analysis, 26:377-381, 2006. 